\title{
Somatotipo, Composición Corporal, Estado Nutricional y Condición Física en Personas con Discapacidad Visual que Practican Goalball
}

\author{
Somatotype, Body Composition, Nutritional State and Physical Condition \\ in People with Visual Impairment Who Practice Goalball
}

\author{
Pablo Antonio Valdés Badilla, ${ }^{* * * *}$; Andrés Esteban Roberto Godoy Cumillaf ${ }^{* * *}$ \& Tomás Nicolás Herrera Valenzuela********
}

VALDÉS, B. P. A.; GODOY, C. A. E. R. \& HERRERA, V. T. N. Somatotipo, composición corporal, estado nutricional y condición física en personas con discapacidad visual que practican goalball. Int. J. Morphol., 32(1):183-189, 2014.

RESUMEN: Las publicaciones referidas a personas con discapacidad visual que practican Goalball son escasas, dado a estudiar otros grupos de la población o bien por razones que se desconocen. El objetivo de esta investigación fue medir el somatotipo, composición corporal, estado nutricional y condición física por aspectos estructurales y funcionales en personas con discapacidad visual que practican Goalball en la región de la Araucanía. El tipo de investigación contempla un diseño no experimental, descriptivo, transversal, con un enfoque cuantitativo. La población estuvo constituida por 14 deportistas de Goalball de la región de la Araucanía, Chile. La muestra fue seleccionada bajo un criterio probabilístico, que incluyo a 11 personas, todos hombres (edad 42,36 $\pm 14,46$ años), en relación al peso y estatura la media fue de 74,76 $\pm 10,36 \mathrm{~kg}$ y 1,695 $\pm 0,07 \mathrm{~m}$, respectivamente. Para medir el somatotipo y la composición corporal se utilizó el protocolo descrito por la Sociedad Internacional para el avance de la Kineantropometría (ISAK) y para evaluar el estado nutricional y la condición física, se utilizaron las pruebas empleadas en el Sistema de medición de la calidad de la educación (SIMCE) para el subsector de Educación Física, propuestos por el Ministerio de Educación de Chile (MINEDUC). Los resultados indican que los deportistas evaluados se clasifican como mesoendomorfos, alcanzando un $28,78 \%$ de masa grasa y $42,71 \%$ de masa muscular, mientras que su IMC fue $26,05 \mathrm{~kg} / \mathrm{m}^{2}$. En cuanto a la condición física los valores encontrados señalan que se encuentran en un nivel satisfactorio para las pruebas de abdominales, flexibilidad y resistencia cardiorrespiratoria, mientras que ostentan malos resultados para las pruebas de salto largo a pies juntos y flexoextensión de codos, situación que se puede entender por la edad, discapacidad y características de la disciplina deportiva practicada.

PALABRAS CLAVE: Somatotipo; Composición corporal; Antropometría; Discapacidad visual; Goalball; Estado nutricional y condición física.

\section{INTRODUCCIÓN}

Las publicaciones referentes al somatotipo, composición corporal, estado nutricional, condición física y rendimiento deportivo son numerosas en el ámbito internacional, las cuales han estudiado a distintos grupos de la población. Sin embargo cuando se busca información relativa a personas con discapacidad visual el espectro se reduce a trabajos que abordan fundamentalmente aspectos sociales y antropométricos (Amorim et al., 2010; França-Freitas \& Gil, 2012; González, 2010; Rodriguez et al., 2007; Santana \& Guillén, 1998; Scherer et al., 2012). Por otra, parte al indagar respecto a la cantidad de personas discapacitadas en
Chile, la Organización Mundial de la Salud (OMS, 2002) y el Servicio Nacional de la Discapacidad (SENADIS, 2010) señalan que un $16 \%$ de la población se encuentra en este grupo, esto quiere decir que uno de cada ocho Chilenos es discapacitado, siendo la región de la Araucanía la segunda con mayor prevalencia del país (INE, 2012).

En relación a las actividades físicas y deportivas que practican las personas con discapacidad visual, la más popular corresponde al Goalball, disciplina Paralímpica creada tras la Segunda Guerra Mundial con la intención de rehabilitar y

\footnotetext{
*entro de Deportes y Salud, Universidad Autónoma de Chile, sede Temuco, Chile.

** Departamento de Educación Física, Facultad de Educación, Universidad Autónoma de Chile, sede Temuco, Chile.

*** Facultad de Ciencias de la actividad física, Universidad San Sebastián, Chile

***** Facultad de ciencias médicas de la Universidad de Santiago de Chile, Chile.
} 
recuperar a las personas afectadas por la guerra (Rodríguez et al.). Este deporte se caracteriza por combinar intervalos de trabajo con pausas para la recuperación y con un tiempo de juego reducido, pero de alta intensidad. Se considera un deporte colectivo, y se identifica por tener relaciones individuales, es decir la relación motriz del jugador con su propio espacio de juego, actuando con gestos técnicos fundamentales en cada fase del partido, tanto en ataque como defensa (Castro et al.). No existe un catastro pormenorizado de las personas que practican Goalball en Chile o bien esta información es desconocida, sin embargo en el ámbito competitivo la región de la Araucanía cuenta con 2 equipos que participan de una liga oficial (Corporación Bartimeo, 2013).

El objetivo de este estudio fue medir el somatotipo, composición corporal, estado nutricional y condición física por aspectos estructurales (abdominales, salto largo pies juntos, flexoextensiones de codos y flexión anterior de tronco) y por aspectos funcionales a través de la prueba de Cafra en personas con discapacidad visual que practican Goalball en la región de la Araucanía.

\section{MATERIAL Y MÉTODO}

El tipo de Investigación contempló un diseño no experimental, descriptivo, transversal, con un enfoque cuantitativo. La población estuvo constituida por 14 deportistas de Goalball de la región de la Araucanía, Chile. La muestra fue seleccionada bajo un criterio probabilístico, que incluyo a 11 personas con impedimentos visuales, nueve son ciegos (tienen su visión clasificados como B1) y dos tienen restos visuales (uno clasificado como B2 y uno como B3), esto de acuerdo a la clasificación oftalmológica de la Federación Internacional de Deportes para Atletas Ciegos (Nascimento \& Morato, 2006). Respecto a la edad, peso, talla y perímetro de cintura se pueden apreciar en la Tabla I la media y desviación estándar (DE) de los deportistas evaluados. Se incluyó a todos los practicantes de Goalball competitivo de la región de la Araucanía, que se encontraban presentes al momento de realizar las pruebas; además debían cumplir con la firma del consentimiento informado, excluyendo a quienes no asistieron, no firmaran el documento solicitado o no quisieron realizar las evaluaciones. El estudio fue desarrollado siguiendo lo expuesto en la Declaración de Helsinki, respecto al trabajo con seres humanos.
Para realizar las evaluaciones, se comenzó con la composición corporal, la cual se fraccionó según el método descrito por Kerr (1988), para el somatotipo se utilizó el método de Heath \& Carter (1967). Las medidas fueron tomadas según el protocolo descrito por la ISAK (MarfellJones et al., 2006). El peso corporal fue evaluado con balanza digital (Scale-tronix, hecho en USA), la estatura con estadiómetro (Seca modelo 220, hecho en Alemania), Los diámetros se evaluaron con antropómetros marca Rosscraft, los perímetros con una cinta métrica marca Sanny y los pliegues cutáneos con el cáliper Harpenden con una precisión de $0,2 \mathrm{~mm}$.

Posteriormente se desarrollaron las pruebas de condición física, las que se realizaron en el Coliseo de la Universidad Autónoma, para ello se comenzó con un calentamiento general de 15 minutos guiado por el Entrenador regional de Goalball. A continuación se dividieron en tres grupos para realizar las pruebas relativas a los aspectos estructurales (abdominales cortos, salto largo a pies juntos, flexo extensión de codos y flexión de tronco adelante); una vez que todos los sujetos pasaban por todas las estaciones mencionadas, se finalizaba con la prueba referida a los aspectos funcionales (prueba de Cafra). De acuerdo a lo expuesto por el MINEDUC (2012) estas pruebas han sido seleccionadas, validadas y estandarizadas nacional e internacionalmente.

En relación a los criterios establecidos en la literatura (Blázquez Sánchez, 2010; López \& Fernández, 2006; Martínez, 2002) para cada una de las pruebas evaluadas, se consideró asociar los puntajes obtenidos por los deportistas de Goalball de la siguiente manera: para la prueba de salto largo a pies juntos se contemplaron los registros que alcanzaron los niveles de 1 al 10, en una escala de 1 a 10 , donde 1 corresponde al nivel más bajo y 10 al más alto; para la prueba de flexo extensión de codos se consideraron los resultados que alcanzaron los niveles de aceptable, bueno y excelente; para la prueba de flexibilidad se estimaron los puntajes que estuvieron dentro de los niveles aceptable, bueno y excelente; en el caso del test de cafra se consideró los registros del pulso al concluir la prueba con la intención de establecer el riesgo cardiovascular de los deportistas. Se utilizaron las categorías señaladas pero en sus valores numéricos para establecer el nivel de satisfactorio.

Tabla I. Descripción de la muestra constituida por deportistas de Goalball de la región de la Araucanía, Chile.

\begin{tabular}{cccccc}
\hline & $\begin{array}{c}\text { Edad } \\
(\mathbf{a n ̃ o s})\end{array}$ & $\begin{array}{c}\text { Peso Corporal } \\
(\mathbf{k g})\end{array}$ & $\begin{array}{c}\text { Estatura } \\
(\mathbf{m})\end{array}$ & $\begin{array}{c}\text { IMC } \\
\left(\mathbf{k g} / \mathbf{m}^{2}\right)\end{array}$ & $\begin{array}{c}\text { Perímetro } \\
\text { cintura (cm) }\end{array}$ \\
\hline $\begin{array}{c}\text { Media } \pm \text { DE } \\
(\mathrm{n}=11)\end{array}$ & $42,36 \pm 14,46$ & $74,76 \pm 10,36$ & $1,695 \pm 0,07$ & $26,05 \pm 3,6$ & $90,52 \pm 8,5$ \\
\hline
\end{tabular}

Para todos los casos se estableció un valor de significancia de $\mathrm{P}<0,05$. 
A continuación se describen cada una de las pruebas realizadas, de acuerdo al MINEDUC:

1.- Estimación del Índice de masa Corporal (IMC): Esta prueba se utiliza para determinar la relación entre el peso y la talla de las personas. El IMC se obtiene dividiendo el peso por la altura al cuadrado $\left(\mathrm{kg} / \mathrm{m}^{2}\right)$.

2.- Perímetro de cintura: Esta prueba se utiliza para estimar la acumulación de grasa en la zona central del cuerpo.

3.- Rendimiento Muscular: Hacen referencia a la capacidad de trabajo de los músculos. Dentro de este componente, se evaluó la fuerza y la resistencia muscular.

3.1.- Abdominales cortos: Esta prueba se utiliza para evaluar la resistencia de la musculatura flexora del tronco.

3.2.- Salto largo a pies juntos: El objetivo de esta prueba es evaluar la fuerza explosiva del tren inferior.

3.3.- Flexo-extensión de codos: Esta prueba se utiliza para medir la resistencia de la fuerza del tren superior.

4.- Flexibilidad: Se define como la capacidad funcional de las articulaciones de moverse en todo su rango de movimiento o bajo la influencia de fuerzas externas, sin dañar músculos ni articulaciones.

4.1.- Flexión de tronco adelante (Wells \& Dillon adaptado): El objetivo de esta prueba es determinar el rango de movimiento de la articulación coxofemoral y de la columna lumbar; determinar la capacidad de elongación de las musculaturas isquiotibial y glútea, y determinar la capacidad flexora de la columna vertebral.

5.- Resistencia Cardiorrespiratoria: Es la capacidad del organismo de suministrar el oxígeno necesario a los músculos y posponer la aparición de la fatiga en una actividad física.
5.1.- Prueba de Cafra: El objetivo de este test es determinar la capacidad adaptativa cardiovascular de las personas a partir de cargas de trabajo de mediana intensidad durante la marcha". Para este estudio se utilizó con la intención de establecer el pulso por minuto (ppm) en un trabajo aeróbico.

Respecto al análisis estadístico, la construcción de la base de datos se realizó con el programa Excel versión 7.0 para ser exportados al programa estadístico GraphPad Prism 5.0 (Graphpad Software, San Diego, CA, USA), las variables fueron sometidas a la prueba de normalidad de Kolmogorov Smirnov (KS) y a un análisis descriptivo (media, DE, valor mínimo y valor máximo). Para las variables que no siguieron un comportamiento normal se determinaron los percentiles 25 y 75. Además se correlacionaron las pruebas de condición física con la edad, peso corporal, estatura, IMC y perímetro de cintura, a través de la pruebas de Pearson y Sperman para las variables paramétricas y no paramétricas, respectivamente. Para todos los casos se estableció un valor de significancia de $\mathrm{P}<0,05$.

\section{RESULTADOS}

En la Tabla II se presentan los valores mínimos, media, DE y máximo de los deportistas de Goalball respecto a la endomorfía, mesomorfía y ectomorfía; reflejando que los evaluados ostentan un somatotipo mesoendomorfo. Enseguida se puede apreciar la Figura 1, que señala la ubicación promedio de la muestra en la somatocarta.

En relación a la composición corporal se presentan los valores de los cinco componentes evaluados, que se pueden observar en la Tabla III, distribuidos en porcentajes mínimos, medios, desviación estándar y máximo de los deportistas estudiados.

En cuanto a los resultados para las variables

Tabla II. Somatotipo de los deportistas de Goalball de la región de la Araucanía, Chile.

\begin{tabular}{lccc}
\hline & Endomorfía & Mesomorfía & Ectomorfía \\
\hline Mínimo & 0,9 & 2,9 & 0,1 \\
Media \pm DE & $4,7 \pm 2,1$ & $5,1 \pm 1,11$ & $1,7 \pm 1,7$ \\
Máximo & 8,4 & 6,9 & 5,9 \\
\hline
\end{tabular}

Tabla III. Composición Corporal de los deportistas de Goalball de la región de la Araucanía, Chile.

\begin{tabular}{lcccccc}
\hline & \% Grasa & \% Músculo & \% residual & \% Ósea & \% Piel* & RCE \\
\hline Mínimo & 20,3 & 31,18 & 9,56 & 8,58 & 4,22 & 0,47 \\
Media \pm DE & $28,78 \pm 8,21$ & $42,71 \pm 5,88$ & $12,85 \pm 2,41$ & $10,78 \pm 1,82$ & $4,88(4,61 ; 4,92)$ & $0,52 \pm 0,03$ \\
Máximo & 45,07 & 52,46 & 16,99 & 13,81 & 6,62 & 0,57 \\
\hline
\end{tabular}

$*=$ Los datos no siguen una curva normal (percentil $25 ; 75$ ). 


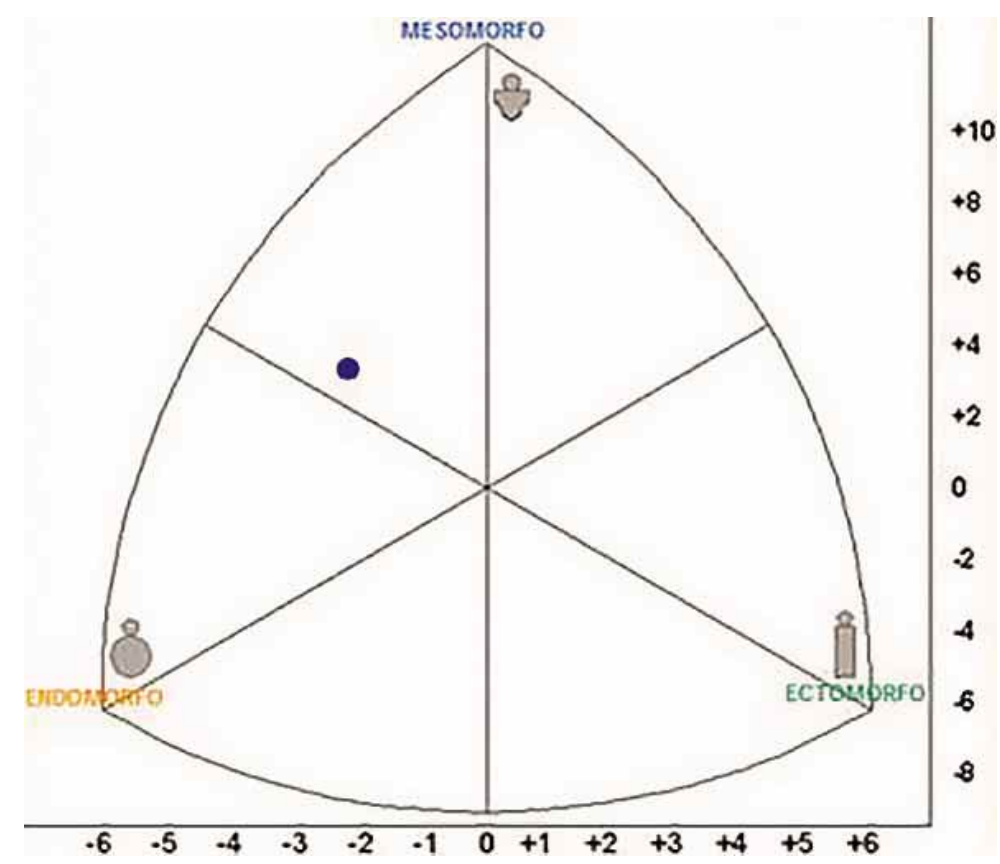

Fig. 1. Somatocarta que muestra la ubicación promedio entre los diferentes somatotipos encontrados en la muestra de deportistas de Goalball de la región de la Araucanía, Chile.

Tabla IV. Variables Antropométricas estimadas en los deportistas de Goalball de la región de la Araucanía, Chile.

\begin{tabular}{|c|c|c|c|}
\hline Variables Antropométric as & Mínimo & Media \pm DE & Máximo \\
\hline \multicolumn{4}{|l|}{ Diámetros } \\
\hline Biacromial & 36,2 & $40,02 \pm 1,61$ & 41,8 \\
\hline Tórax Transverso & 28 & $30,31 \pm 1,35$ & 32,5 \\
\hline Tórax Antero-posterior* & 18 & $23,55(19,8 ; 23,4)$ & 39,8 \\
\hline Bi-iliocrestídeo* & 24 & $28,48(28,2 ; 30)$ & 30,6 \\
\hline Humeral (biepicondilar) & 6,7 & $6,96 \pm 0,2$ & 7,3 \\
\hline Femoral (biepicondilar) & 9,2 & $9,64 \pm 0,31$ & 10,2 \\
\hline \multicolumn{4}{|l|}{ Perímetros } \\
\hline Cabeza* & 52,9 & $55,76(55 ; 56,7)$ & 57,8 \\
\hline Brazo relajado & 24 & $30,36 \pm 2,97$ & 35 \\
\hline Brazo flexionado en tensión & 29 & $32,11 \pm 2,58$ & 36,5 \\
\hline Antebrazo Máximo & 24 & $26,82 \pm 1,31$ & 28,5 \\
\hline Tórax Mesoesternal* & 87 & $100,8(100,5 ; 104)$ & 107 \\
\hline cintura (mínima) & 74 & $90,02 \pm 8,73$ & 103 \\
\hline cadera (máximo)* & 71 & $93,47(90 ; 99)$ & 108 \\
\hline Muslo (máximo) & 44 & $57,34 \pm 11,18$ & 86,2 \\
\hline Muslo (medial) & 43 & $50,87 \pm 4,95$ & 60 \\
\hline Pantorrilla (máxima) & 30 & $35,57 \pm 2,45$ & 38 \\
\hline \multicolumn{4}{|l|}{ Pliegues Cutáneos } \\
\hline Tríceps & 8,8 & $14,13 \pm 6,04$ & 28,5 \\
\hline Subescapular & 10 & $22,92 \pm 10,54$ & 42 \\
\hline Supraespinal & 6,2 & $15,64 \pm 7,51$ & 30 \\
\hline Abdominal* & 10 & $29,92(20,15 ; 42)$ & 55 \\
\hline Muslo & 7 & $17,26 \pm 12,44$ & 49 \\
\hline Pantomilla & 5 & $10,66 \pm 6,31$ & 25 \\
\hline
\end{tabular}

*= Los datos no siguen una curva normal (percentil 25; 75). antropométricas, se han expresado en media, DE, mínimo y máximo. Dichas medidas corresponden a los diámetros, perímetros y pliegues cutáneos de los deportistas de Goalball, estos registros se pueden apreciar en la Tabla IV.

El estado nutricional de los sujetos, se obtuvo a través del IMC, los que se presentan en porcentaje; mientras que su categorización se expresa en; bajo peso, normal, sobrepeso y obesidad. Resultados que se pueden observar en la Tabla V.

Respecto a la condición física de la población estudiada se aprecia en la Tabla VI los resultados obtenidos para las pruebas de flexibilidad, salto largo a pies juntos, abdominales, flexo extensión de codos y test de cafra expresados en media y desviación estándar. Mientras que en la Tabla VII se pueden observar los porcentajes alcanzados por los deportistas al clasificarlos como satisfactorio y no satisfactorio, de acuerdo a lo propuesto por el MINEDUC.

\section{DISCUSIÓN}

El somatotipo de los atletas evaluados presentaron un promedio de 4,7 - 5,1 - 1,7, valores que difieren a los registrados por Scherer et al., quien evaluó a un atleta de la selección Brasilera de Goalball, con valores de 1,5 - 5,5 2,8. En tanto, Carter \& Heath (1990) plantean que el somatotipo es fenotípico, y por ello es susceptible a cambios, producidos entre otras cosas, por el entrenamiento. Si analizamos estos valores, vemos que el atleta paralímpico, tiene un promedio de 12 horas de entrenamiento semanal, mientras que los atletas estudiados, informan dedicar solo 3 horas semanales, situación que respalda los promedios obtenidos para el IMC $(26,05)$, el cual difiere al encontrado en el seleccionado Brasileño (23,04).

En relación a la composición corporal, los valores de masa grasa y masa muscular para los atletas evaluados alcanzan un promedio de $28,78 \%$ y $42,71 \%$ respectivamente. De acuerdo a lo expuesto por Scherer et al., los valores obtenidos por el atleta paralímpico estudiado alcanza un $11,49 \%$ de masa grasa y $45,51 \%$ de masa muscular. En relación a la masa muscular, 
Tabla V. Distribución porcentual del estado nutricional en los deportistas de Goalball de la Región de la Araucanía, Chile.

\begin{tabular}{lcccc}
\hline \multicolumn{5}{c}{ Porcentaje de Índice de masa Corporal (IMC) } \\
\hline Porcentaje & Bajo Peso & Normal & Sobrepeso & Obesidad \\
$\mathbf{n = 1 1}$ & 0 & $45,45 \%$ & $36,36 \%$ & $18,18 \%$ \\
\hline
\end{tabular}

Tabla VI. Media y desviación estándar de la condición física de los deportistas de Goalball de la Región de la Araucanía, Chile.

\begin{tabular}{cccccc}
\hline & Flexibilidad & Salto largo & Abdominales & Flexo extensión de & Test de Cafra \\
\hline $\begin{array}{c}\text { Media } \pm \text { DE }(\mathbf{n} \\
=\mathbf{1 1})\end{array}$ & $31,82(25 ; 35)$ & $150,5 \pm 37,65$ & $24,36(25 ; 25)$ & $20,45(15 ; 22)$ & $111,8 \pm 25,23$ \\
\hline
\end{tabular}

*= los datos que no presentan una distribución normal son presentados en media (percentil 25; percentil 75). Solamente se encontró una correlación positiva entre la estatura y la prueba de salto largo a pies juntos $(r=0,678 ; \mathrm{P}<0,05)$, sin encontrar relación entre el resto de las variables.

Tabla VII. Distribución porcentual de la condición física en los deportistas de Goalball de la Región de laAraucanía, Chile.

\begin{tabular}{ccccccccccc}
\hline & \multicolumn{2}{c}{ Flexibilidad } & \multicolumn{2}{c}{ Salto largo } & \multicolumn{2}{c}{ Abdominales } & \multicolumn{2}{c}{$\begin{array}{c}\text { Flexo extensiones } \\
\text { de codos }\end{array}$} & \multicolumn{2}{c}{ Test de Cafra } \\
\hline Porcentaje & S & NS & S & NS & S & NS & S & NS & S & NS \\
$\mathbf{n = 1 1}$ & 63,64 & 36,36 & 0 & 100 & 90,91 & 9,90 & 27,27 & 72,73 & 90,91 & 9,09 \\
\hline
\end{tabular}

$\mathrm{S}=$ Satisfactorio NS $=$ No Satisfactorio.

no hay grandes diferencias, pero al comparar los valores de masa grasa se encuentran divergencias. Esto podría explicarse por lo descrito por Martins \& Rodríguez Dos Santos (2004), quienes plantean que la práctica regular de actividad física provoca una baja en los niveles de grasa corporal, por ello el atleta paralímpico al dedicar más horas al entrenamiento logra valores más favorables respecto a los deportistas de la Araucanía.

Pazmiño \& Sánchez (2011) plantean que las personas con discapacidad visual son dependientes de otros para moverse, limitando su participación en actividades que los beneficie y situándolos mayormente en el grupo de personas sedentarias. Sustento que da respaldo a lo encontrado respecto al estado nutricional, ya que sólo el $45,45 \%$ de los deportistas evaluados se halla en el rango normal y el 54,54\% tiene sobrepeso u obesidad.

$\mathrm{Al}$ indagar en torno a las pruebas de condición física se halla el trabajo realizado por Amorim et al., quienes analizaron la dinámica del Goalball y señalan que se utilizan fundamentalmente acciones de lanzamientos, deslizamientos y posición arrodillado, lo que explica los malos resultados alcanzados en la prueba de salto largo a piesjuntos (150,5 $\mathrm{cm})$, circunstancia que se respalda con lo expuesto por Martínez al plantear que la reducción de experiencias motrices en las personas no videntes, contribuyen al retraso en una acción motriz determinada.
Además, según Amorim et al., no se encuentra actividad alguna en el Goalball donde esté implícita la flexo extensión de codos, que junto a lo planteado por Urzanqui (2007), que las personas con discapacidad visual tienen varios grupos musculares hipotróficos producto de una menor utilización de éstos, pueden entenderse los resultados obtenidos por los sujetos evaluados quienes se clasifican mayormente como no satisfactorios para la prueba de flexo extensión de codos $(72,73 \%)$.

Respecto a la prueba de flexibilidad, un $63,64 \%$ se encuentra en la clasificación de satisfactorio, debido entre otras cosas, a que esta es una capacidad que se trabaja repetidamente, tanto para lograr una mayor amplitud de los movimientos realizados, como para prevenir posibles lesiones de un proceso de entrenamiento competitivo (Scherer et al.).

En la prueba de abdominales cortos, tambien se clasifican como satisfactorios un $90,91 \%$ de los evaluados, debido entre otros factores a que este grupo muscular es el que trabaja constantemente durante el juego al ejecutar una de las acciones fundamentales que tiene el deporte, la cual es pasar de la posición extendido en la defensa, a la de arrodillado en el ataque, ejercicio que se realiza repetidamente durante el entrenamiento y los partidos.

En cuanto a los resultados para la prueba de resistencia cardiorrespiratoria, en particular para la prueba de Cafra, 
los evaluados alcanzaron mayormente el nivel satisfactorio $(90,91 \%)$, lo que se entiende por lo expuesto por Urzanqui, quien habla de los beneficios que trae la práctica del Goalball en personas ciegas, entre ellos se encuentra el de un mayor aporte de oxígeno y una utilización periférica más efectiva de este, mejorando la capacidad cardiovascular y respiratoria, dando cuenta de la adaptación que reflejan los deportistas con el trabajo aeróbico.

\section{CONCLUSIÓN}

Los resultados encontrados en nuestra investigación dan cuenta de buenos niveles de somatotipo y de composición corporal respecto a la masa muscular, en tanto que los registros para la masa grasa e IMC deben mejorarse. En relación a la condición física se puede indicar que los deportistas de Goalball alcanzan valores favorables para las pruebas de abdominales, flexibilidad y resistencia cardiorrespiratoria, mientras que obtienen bajos resultados para las pruebas de salto largo a pies juntos y flexoextensión de codos, situación que se puede entender por la edad, discapacidad y características de la disciplina deportiva practicada.

Es relevante ahondar en este tipo de investigaciones que involucra a un grupo de la población poco estudiado y que demuestra mayor independencia en el quehacer diario con la práctica deportiva. Se requiere un mayor número de estudios en deportistas que practiquen Goalball con el objetivo de establecer el perfil físico y funcional del jugador.

\section{AGRADECIMIENTOS}

Al presidente de la Corporación Bartimeo, Sr. Rodrigo Lincoleo, al Profesor Leonel Quintana y a todos los jugadores de Goalball de la región de la Araucanía que participaron de la Investigación.

VALDÉS, B. P. A.; GODOY, C. A. E. R. \& HERRERA, V. T. N. Somatotype, body composition, nutritional state and physical condition in people with visual impairment who practice goalball. Int. J. Morphol., 32(1):183-189, 2014.

SUMMARY: Few publications refer to people with a visual impairment who practice Goalball, given that other groups of the population were studied or for unknown reasons. The aim was to measure the somatotype, the body composition, the nutritional state, and the physical condition (more specifically structural and functional features) of people with visual impairment in the Araucanía Region in Chile. The type of investigation considers a cuantitative, non-experimental, descriptive and transversal design. The sample consists of 14 Goalball athletes of the Araucanía Region. The sample was selected randomly and included 11 male subjects (age $42.36 \pm 14.46$ ), with respect to weight and height the average was $74.76 \pm 10.36$ and $1.695 \pm 0.07$ respectively. To measure the somatotype and the body composition, the protocol described by the International Society for the Advancement of Kinanthropometry (ISAK) was used, whereas to evaluate the nutritional state and physical condition, the tests employed in the SIMCE (System of Measuring the Quality of Education) for the subsector of Physical Education in Chile (MINEDUC - Ministry of Education) were administered. The results indicate that the evaluated athletes classify as meso-endomorph. They reached $28.78 \%$ of fat mass and $42.71 \%$ of muscle mass, whereas their BMI is $26.05 \mathrm{~kg} / \mathrm{m}^{2}$. Regarding their physical condition the findings show a satisfactory level for the tests of abdominals, flexibility, and cardiorespiratory resistence. The tests of long jump with joined feet and elbow-flexo-extention were not as good, however. These outcomes can possibly be explained by the age, visual impairment, and by characteristics of the sport discipline practiced.

KEY WORDS: Somatotype; Body composition; Anthropometry; Visual impairment; Goalball; Nutritional state and physical condition.

\section{REFERENCIAS BIBLIOGRÁFICAS}

Amorim, M.; Botelho, M.; Sampaio, E.; Molina, J. \& Corredeira, R. Caracterización de los patrones comportamentales de los atletas con discapacidad visual practicantes de Goalball. REIFOP, 13(3):47-57, 2010.

Blázquez Sánchez, D. Evaluar en Educación Física. Barcelona, INDE, 2010.

Carter, J. E. L. \& Heath, B. H. Somatotyping-development and applications. Cambridge, Cambridge University Press, 1990.
Corporación Bartimeo. Informe $2^{\circ}$ fecha Nacional de Goalbal. Temuco, Corporación Bartimeo, 2013.

França-Freitas, M. L. P. \& Gil, M. S. C. A. Interação social de crianças cegas e de crianças videntes na educação infantil. Psicol. Esc. Educ., 16(2):317-27, 2012.

González, S. E. Necesidades Educativas especiales en Educación Física. Revista Digital Innovación y Experiencias Educativas, 36:50-62, 2010. 
Heath, B. H. \& Carter, J. E. A modified somatotype method. Am. J. Phys. Anthropol., 27(1):57-74, 1967.

INE. Informe, Instituto Nacional de estadísticas, 2012. Disponible en: http://www.ine.cl/

Kerr, D. An anthropometric method for fractionation of skin, adipose, bone, muscle and residual masses in males and females age 6 to 77 years. M.Cs. Kinesiology Tesis, British Columbia, Simon Fraser University, 1988.

López, C. \& Fernández, V. Fisiología del ejercicio. 3a ed. Madri, Panamericana, 2006.

Marfell-Jones, M.; Olds, T.; Stewart, A. D. \& Carter, L. International Standards for Anthropometric Assessment. Potchefstroom, International Society for the Advancement of Kinanthropometry (ISAK), 2006. pp.61-75.

Martínez, O. L. Condición física y nivel de actividad física en estudiantes universitarios. Revista Teoría y Praxis Investigativa, 3(1):21-8, 2002.

Martins, F. R. \& Rodríguez Dos Santos, J. A. Atividade fisica de lazer, alimentaçao y composiçao corporal. Rev. Bras. Educ. Fís. Esp., 18(2):159-67, 2004.

MINEDUC. SIMCE 2012 Educación Física, resultados para Docentes y Directivos. Santiago, Ministerio de Educación, 2012.

Nascimento, D. \& Morato, M. Manual de orientação para professores de Educação física: Goalball. Brasília, Comitê Paraolímpico Brasileiro, 2006.

OMS. Informe sobre la salud en el mundo. Ginebra, Organización Mundial de la Salud, 2002.

Pazmiño, A. \& Sánchez, D. La condición física en niños (as) con capacidades especiales de la escuela Geovanny Calle de la ciudad de Cayambe. Realización de una metodología de test para el atletismo orientado a olimpiadas especiales 2011 . Tesis para optar al Título de Licenciado en la especialidad de Entrenamineto deportivo. Ibarra, Ecuados, Universidad Técnica del Norte, 2011.

Rodriguez, A.; García, A. \& Caurcel, M. La práctica de la actividad física y deportiva en el alumnado invidente o con deficiencia visual. En: Educación, Actividad física, Salud y Empresa. Educación para la salud, prevención y gestión de recursos. Melilla, Centro UNESCO de la Ciudad Autónoma de Melilla, 2007. pp.46-55.

Santana, A. \& Guillén, F. El abandono deportivo de personas con deficiencia visual. Área de Psicología del Deporte y Control Motor - Rendimiento Deportivo. I Congreso de la Asociación Española de Ciencias del Deporte, 1998. pp.359-66.

Scherer, R.; Karasiak, F.; Silva, S. \& Petroski, E. Morphological profile of goalball athletes. Mot. Eur. J. Hum. Mov., 28:1-13, 2012.

SENADIS. Resultado Nacional Prevalencia de personas con discapacidad en Chile. Santiago, Servicio Nacional de la Discapacidad, 2010.

Urzanqui, A. Beneficios del ejercicio físico y el deporte para la salud en ciegos y deficientes visuales. Segunda conferencia internacional sobre deporte adaptado. Fundación Andalucía olímpica, II Conferencia Internacional sobre Deporte Adaptado, Málaga, 15-17 de Marzo, 2007.

Dirección para Correspondencia

Pablo Antonio Valdés Badilla

Universidad Autónoma de Chile

Porvenir 649

Temuco

CHILE

Email: pablo.valdes@uautonoma.cl

Recibido: 14-09-2013

Aceptado: 29-11-2013 